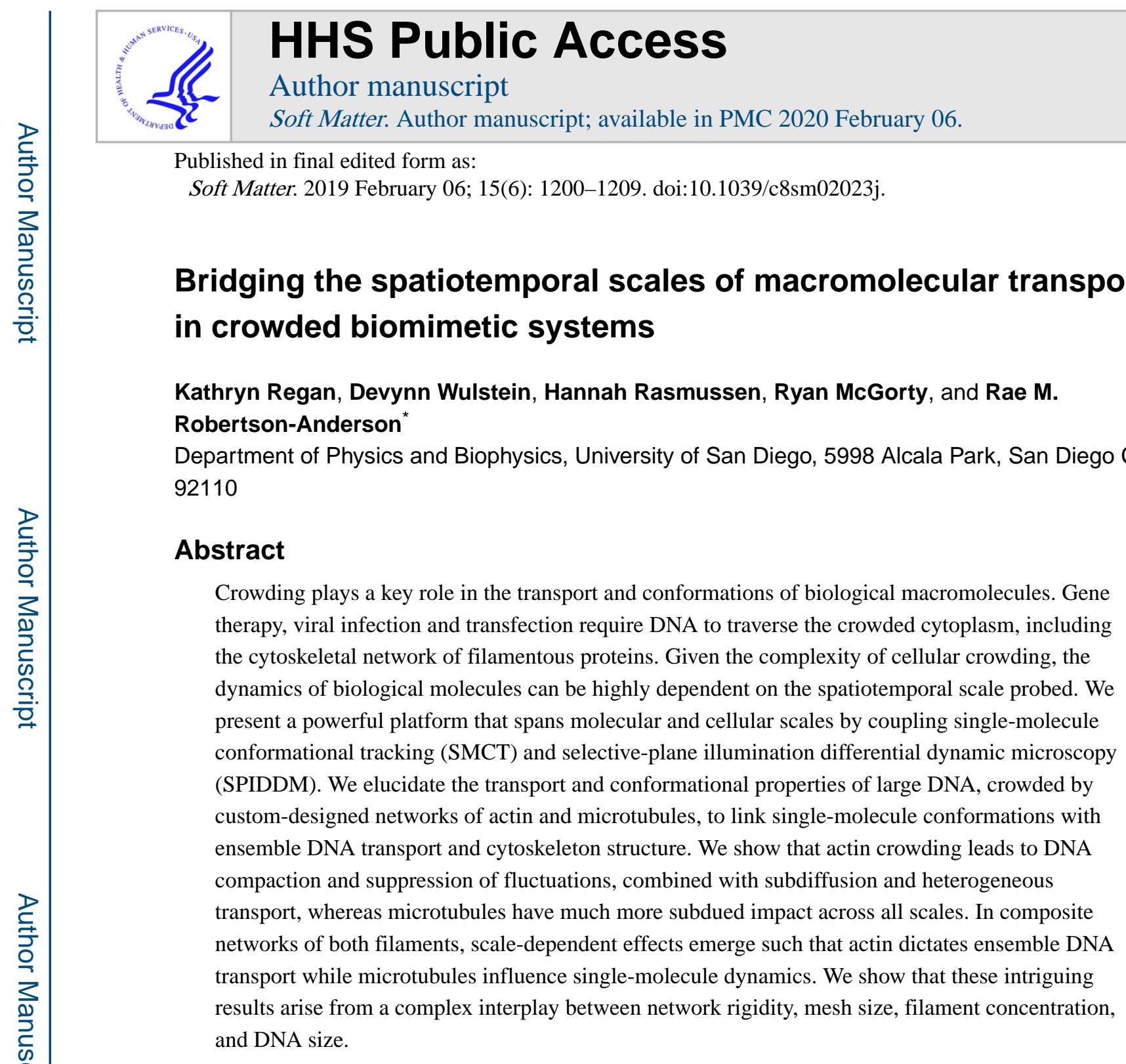

\title{
Graphical Abstarct
}

"Corresponding author.

Conflicts of Interest

The authors have no conflicts to declare. 

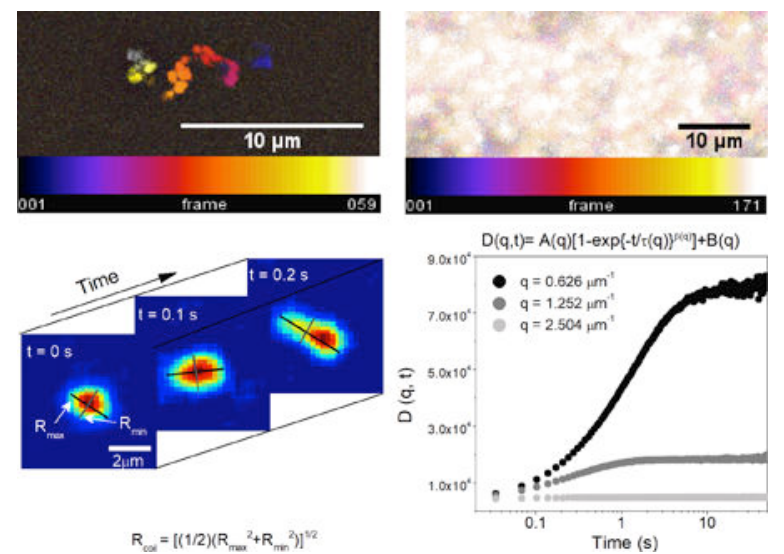

\section{Introduction}

The impact of crowding on macromolecular transport and conformations is a topic of intensive theoretical and experimental research. ${ }^{1-9}$ The primary inspiration for much of this work is to understand how large biomacromolecules such as nucleic acids and proteins can function in the highly crowded environment of the cell. For example, DNA transport through the crowded cytoplasm is essential for transformation, transcription, looping, gene expression, viral infection and gene therapy. $1,2,9,10$ Crowding is arguably the most important factor in determining passive intracellular transport properties and also plays a key role in determining the conformations, and thus stability and function, of macromolecules.

Most in vitro studies aimed at understanding the role that crowding plays in macromolecular conformations or transport have focused on the effect of small soluble proteins, often mimicked with synthetic crowders such as dextran or PEG. ${ }^{3,11-14}$ Several of these studies have reported normal Brownian motion of spherical tracers and DNA, in which the meansquared displacement in each direction (MSD) scales linearly with time (i.e. $M S D=2 D t$ where $D$ is the diffusion coefficient). ${ }^{15-18}$ However, others have found anomalous subdiffusion where $M S D=K t^{\alpha}$ with $K$ being the transport coefficient and $a$ being in the range of $\sim 0.4-0.9 .^{4,19-21}$ These studies have also shown evidence of macromolecular compaction, swelling, and elongation depending on the type and size of tracer macromolecule and crowder. ${ }^{4,5,22}$ These crowding-induced conformational changes in turn impact the resulting transport properties.

Much less appreciated is the fact that the cytoplasm of eukaryotic cells is also crowded by pervasive networks of filamentous proteins that comprise the cytoskeleton. ${ }^{23,24}$ Given the size and rigidity of cytoskeletal proteins, the steric interactions between them, and the viscoelastic nature of the networks, the cytoskeleton presents a key barrier to efficient gene delivery, and its impact on DNA transport is likely highly unique to that of the widelystudied viscous systems of soluble proteins. ${ }^{4,15,19,25}$ Two ubiquitous cytoskeletal proteins are semiflexible actin filaments, with a persistence length of $l_{p} \sim 10 \mu \mathrm{m}$ and thickness of $\sim 7$ $\mathrm{nm}$, and rigid microtubules, with $I_{p} \sim 1 \mathrm{~mm}$ and thickness of $\sim 25 \mathrm{~nm} .{ }^{4,23,26,27}$ At physiologically relevant concentrations, both filaments exhibit length distributions of $\sim 1-$ $50 \mu \mathrm{m}$ and form sterically interacting networks with mesh sizes of $\sim 0.1-1 \mu \mathrm{m}$. Intermediate 
filaments, with thickness in between that of actin and microtubules, also contribute to cytoskeleton structure, but are far less dynamic and rigid. ${ }^{23}$ The resulting cytoskeletal environment can thus lead to highly heterogeneous transport of macromolecules over a range of length and time scales.

This inherent complexity, similar to other biological networks such as mucus and the extracellular matrix, limits the ability of standard particle-tracking and scattering methods to independently gather a comprehensive picture of macromolecular transport dynamics within these networks. For example, measurements of nanospheres diffusing in actin networks have reported diffusion coefficients that vary over 2 orders of magnitude depending on the timescale probed. ${ }^{28}$ Similar studies have reported subdiffusion in actin networks at intermediate times but normal diffusion at short and long times. ${ }^{29}$ These results highlight the importance of measurements with fast time resolution and long timescales. This effect is amplified when examining environments composed of more than one crowder species, where the range of dynamics can be even more spread out in time and space. ${ }^{30}$ Singlemolecule tracking can quantify the distributions of conformational dynamics and trajectories of single diffusing macromolecules. However, these methods are limited by a need for a large ensemble of molecules to accurately determine MSDs and can often only measure transport over relatively short length and timescales due to photobleaching and diffusion beyond a limited region-of-interest. Conversely, scattering methods, which investigate bulk patterns in diffusion, can measure transport properties over large spatiotemporal scales but are unable to discern individual molecular trajectories or conformations.

Here we present a powerful platform that combines single molecule conformational tracking with digital Fourier microscopy to allow for the coupling of crowding-induced conformations and trajectories of single molecules with ensemble-averaged transport properties across a large range of length and time scales $(\sim 0.1-60 \mathrm{~s}, \sim 0.1-25 \mu \mathrm{m})$ (Fig. 1). The wide spatiotemporal range and variety of measurement deliverables is made possible by integrating two recently developed complementary techniques: selective-plane illumination differential dynamic microscopy (SPIDDM) ${ }^{31}$ and single-molecule conformational tracking (SMCT). $)^{3,6,32}$

SPIDDM couples light-sheet microscopy (LSM), an optical sectioning microscopy technique that allows for minimum exposure to excitation light, with differential dynamic microscopy (DDM), a type of digital Fourier microscopy that quantifies intensity fluctuations within a time series of images. ${ }^{33-36}$ Importantly, DDM determines transport properties over large fields-of-view without localizing or tracking individual DNA molecules, providing access to long times ( $60 \mathrm{~s})$ and large lengthscales $(\sim 25 \mu \mathrm{m})($ Fig. 1$){ }^{31}$ To complement this efficient extraction of ensemble transport dynamics and extend the spatiotemporal range of measurements, we use SMCT to resolve the position, size and shape of individual molecules over time. SMCT is capable of measuring single-molecule conformational fluctuations as small as $\sim 100 \mathrm{~nm}$ with temporal resolution of $0.1 \mathrm{~s}$. These conformational measurements include the time-resolved distributions of major and minor axis lengths $\left(R_{\max }, R_{\min }\right)$ and the spatiotemporal scales over which conformational fluctuations occur (Fig. 1). 
Using this combined approach, we characterize the dynamics of large naked DNA molecules diffusing in custom-engineered cytoskeletal networks, allowing for direct coupling of DNA diffusivity and conformational dynamics with the properties of the surrounding protein networks. In order to model the cytoskeleton, we create tunable, well-characterized networks of actin filaments and microtubules, as well as a composite of both proteins (Fig. 2). ${ }^{30}$ Ensemble analysis reveals that DNA transport in actin and composite networks is highly heterogeneous as well as subdiffusive, whereas microtubule networks induce more homogenous and less restricted transport. SMCT analysis reveals that actin networks appreciably compact DNA and suppress conformational fluctuations, while microtubule and composite networks result in much less compaction with less constrained fluctuations. These results demonstrate the integral role that coupling dynamics across a wide range of scales plays in characterizing macromolecular dynamics in crowded and complex systems. Our presented platform offers a robust route for researchers to efficiently probe macromolecular transport and conformational properties across scales in a vast array of crowded and composite biomimetic networks.

\section{Results and Discussion}

We use SMCT to determine the effect of cytoskeleton network confinement on the conformational dynamics of single DNA molecules (Fig. 1, Methods). ${ }^{3,6,32}$ Specifically, we evaluate the distribution of shapes and sizes of molecular conformations and determine how the measured conformations change over time. The distributions of DNA coil sizes $R_{\text {coil }}=\left[1 / 2\left(R_{\max }{ }^{2}+R_{\text {min }}{ }^{2}\right)\right]^{1 / 2}$ for each condition clearly show that all cytoskeleton networks induce some degree of DNA compaction compared to buffer conditions (Fig. 3A). The degree of compaction is highest for actin filaments while microtubules and composite networks induce comparatively less compaction.

From the $R_{\text {coil }}$ distributions we evaluate the average size, $\left\langle R_{\text {coil }}\right\rangle$, as well as the breadth of sizes accessed by the ensemble of molecules over time, quantified by the standard deviation of coil sizes $\Delta R_{\text {coil }}$ (Fig. 3B). As shown, while $\Delta R_{\text {coil }}$ decreases as $R_{\text {coil }}$ decreases, the ratio $\left.\Delta R_{\text {coil }}<R_{\text {coil }}\right\rangle$, which quantifies the relative breadth of size distributions, is smaller in cytoskeleton networks compared to buffer conditions. This result indicates that cytoskeleton networks not only compact DNA but that these compact conformations are more ordered and exhibit less conformational heterogeneity. Further, actin induces appreciably more compaction than microtubules and composites. A similar effect is apparent when evaluating the DNA shape or eccentricity, which we quantify by comparing $\left\langle R_{\max }\right\rangle$ to $\left\langle R_{\min }\right\rangle$ (Fig. 3C). For reference, $\left\langle R_{\max }\right\rangle:\left\langle R_{\min }\right\rangle$ is 1 for a spherical conformation, $\sim 1.3$ for a random coil, ${ }^{37,38}$ and $>1.3$ for elongated conformations. As shown, all cytoskeleton networks induce a slight shift from random coil eccentricity to sphericity. Fig. $3 \mathrm{C}$ also corroborates that actin networks induce the most extreme compaction, with a similarly more spherical aspect ratio, whereas microtubules and composites induce less extreme effects on size and shape.

Previous experiments on large DNA crowded by synthetic polymers have also reported molecular compaction, coupled with a shift to sphericity and reduced breadth of size distributions, which was attributed to the well-known depletion interaction in crowded 
systems. ${ }^{6,39,40}$ Namely, to maximize the volume available to small crowders, and thus maximize their entropy, large macromolecules are forced to condense to reduce the excluded volume or 'depletion zone' that their contours impose. The degree of compaction or volume reduction has been reported to depend on the shape, structure, and concentration of crowders. $., 8,15,17,39,41-46$ While the compaction we measure likely arises from a similar effect, our results are the first, to our knowledge, to report on depletion-driven compaction in highly entangled, viscoelastic systems or when the crowders are comparable in size or larger than the compacted polymer. The varying degree of compaction in different networks that are at equal molarity further indicates the important role that molecular entanglements and stiffness can play in the excluded volume effect.

The pressure leading to this depletion-driven compaction can be understood in terms of osmotic pressure $p=c k_{B} T$ where $c$ is the number concentration of crowders, $k_{B}$ is the Boltzmann constant, and $T$ is temperature. ${ }^{47}$ While the monomer concentration is the same for all networks, the relevant concentration here is that of freely floating particles, i.e. the filaments themselves. Using actin and tubulin monomer sizes ${ }^{23,48}$ and the measured average lengths of each filament in our networks $\left(L_{A}=8.7 \mu \mathrm{m}, L_{M}=18.8 \mu \mathrm{m}\right),{ }^{30}$ we compute $p$ values of $\sim 9,0.6$ and $5 \mathrm{mPa}$ for actin networks, composites and microtubule networks, respectively. The larger pressure from the actin network compared to microtubules and composites is qualitatively in line with the stronger compaction we see in actin networks. To determine if the pressure is sufficient to compact the DNA we compute the force $F=p \pi R_{\text {coil }}{ }^{2}$ exerted on a sphere of radius $1 / 2 R_{\text {coil }}$, and determine the fractional deformation $x_{f}$ possible according to the worm-like chain model: $F l_{p} / k_{B} T=1 / 4\left(1-x_{f}\right)^{-2}-1 / 4+x_{f} \cdot{ }^{49}$ We find that the force is sufficient to extend or compact the DNA by $x_{f}=39 \%, 25 \%$, and $4 \%$ for actin, composites, microtubules, respectively. Thus, depletion interactions are indeed sufficient to compact the DNA in all networks, and, corroborating our results, compaction should be strongest in actin networks, followed by composites and microtubules.

To determine the time-dependence of conformational states and their dependence on the crowding network, we track the change in $R_{\max }$ over different lag times $t$, termed the fluctuation length $L(t)=\left\langle\left|R_{\text {max }}(0)-R_{\text {max }}(t)\right|\right\rangle$ (Fig. 3D). $L(t)$ quantifies the scales over which DNA molecules fluctuate between different conformational states. As shown, in all conditions $L(t)$ increases with time from an initial value $L_{O}$ to a final, near steady-state value $L_{f}$. However, the extent to which $L(t)$ increases from $L_{0}$, as well as the rate at which it increases, is highly dependent on the type of cytoskeletal network. In agreement with our compaction results, actin networks result in the smallest $L_{O}$ and $L_{f}$ values, while composite crowding induces conformational dynamics closer to that of microtubules (Fig. 3E). To quantify how quickly DNA fluctuates between different conformational states, we calculate the time $\tau_{f}$ needed for $L(t)$ to reach $90 \%$ of its final value $L_{f}, 0.9 L_{f}$ (Fig. 3F). Interestingly, the fluctuation rate $\tau_{f}^{-1}$ increases proportionally with increasing $0.9 L_{f}$, meaning networks that induce more slowing of conformational dynamics also suppress the lengthscale over which molecular conformations fluctuate. Actin induces the most extreme slowing, proportionally suppressing the maximal length of fluctuations, and microtubules have the 
least slowing effect compared to buffer conditions. Composites induce fluctuation rates and lengths intermediate between actin and microtubules.

To couple these intriguing single-molecule conformational dynamics with network-wide analysis of the collective motility of the DNA molecules, we perform DDM analysis (Fig. 1, Methods). By comparing the image structure function $D(q, t)$ for each condition, it is clear that actin networks and composites drastically slow DNA transport, as evidenced by the slow rise in $D(q, t)$ compared to buffer conditions and microtubule networks, as well as the lack of any convergence or plateau at long times (Fig. 4A). Similar to the conformational results described above, microtubules have less of an effect on DNA transport, albeit slowing is still evident. However, crowding by composites appears to have nearly indistinguishable effects on $D(q, t)$ from that of actin, whereas single-molecule DNA conformations in composites appear to be determined largely by microtubules.

We determine the characteristic decay time $\tau$ of density fluctuations of lengthscale $2 \pi q^{-1}$ by fitting $D(q, t)$ to a function containing a stretched exponential (Fig. 4B, Methods). As shown, cytoskeletal networks slow transport (i.e. increase $\tau$ ) across all lengthscales. However, actin and composites exhibit much more pronounced slowing compared to microtubules and similar $\tau(q)$ values across nearly all scales. To further quantify transport, we fit $\tau(q)$ to the power-law function $\tau(q)=\left(K q^{2}\right)^{-1 / a}$, where $a$ and $K$ are the same as in the diffusion equation $M S D=K t^{\alpha}$. Actin and composite networks result in the smallest $K$ values, indistinguishable from one another within error (Fig. 4C). Furthermore, both networks also result in highly subdiffusive transport of DNA ( $a_{\text {actin }} \approx 0.5, a_{\text {composite }} \approx 0.66$ ), whereas the deviation from 1 is weaker for microtubules $(a \approx 0.8)$.

Subdiffusion reported in a wide variety of crowded systems has been attributed to phenomena such as excluded volume, fractional Brownian motion (FBM), viscoelasticity and caging. ${ }^{4,21,41,50}$ Unlike our results, subdiffusion resulting from small mobile crowders is often only apparent over a limited temporal range. ${ }^{21}$ This transitional subdiffusion, which has been reported for small proteins in live cells, has been shown to be a result of excluded volume and FBM. ${ }^{21,41}$ These studies also reported that the apparent transient anomalous transport persisted even with the destruction of the cytoskeleton. As our results seem to be at odds with these previous studies, our observed subdiffusive transport is likely the result of caging and/or viscoelasticity rather than excluded volume or FBM.

Experiments on small DNA fragments $(<3 \mathrm{kbp})$ in cytoplasm and actin networks showed that the presence of an intact actin network was essential to extreme reductions in DNA diffusion coefficients. ${ }^{15,16}$ However, unlike our results, normal diffusion $(a=1)$ was reported for all cases. The notable difference between these studies and ours is the $\sim 40 x$ difference in DNA length ( $<3$ vs $115 \mathrm{kbp}$ ). This size difference, which correlates to a $\sim 6 x$ difference in DNA coil radius, is of principal importance, as the degree of crowding-induced subdiffusion has been shown to depend directly on the ratio of particle or polymer radius to the correlation length of the crowding network. ${ }^{50,51}$ In particular, for colloids diffusing in actin networks, subdiffusion was only apparent when the colloid radius $a$ was comparable to the mesh size of the actin network $\xi_{A}$, with $a$ decreasing linearly with increasing ratios 
$a: \xi_{A} \cdot{ }^{50}$ In our experiments, the DNA coil radius is $\sim 1 / 2 R_{\text {coil }} \approx 0.6-0.8 \mu \mathrm{m}$. The mesh sizes for actin and microtubule networks can be calculated from the corresponding protein concentrations $c_{A}$ and $c_{T}$ via $\xi_{A}=0.3 /\left(c_{A}{ }^{1 / 2}\right)$ and $\xi_{M}=0.89 /\left(c_{T}^{1 / 2}\right)$ where $\xi$ and $c$ are in units of $\mu \mathrm{m}$ and $\mathrm{mg} / \mathrm{ml},{ }^{30,52-54}$ giving values of $\xi_{A} \approx 0.4 \mu \mathrm{m}$ and $\xi_{M} \approx 0.9 \mu \mathrm{m}$, respectively for the networks studied here. ${ }^{52-54}$ In actin-microtubule composites, the corresponding mesh sizes are $\xi_{M}=1.1 \mu \mathrm{m}$ and $\xi_{A}=0.60 \mu \mathrm{m}$, and the effective mesh size is $\xi_{C}=\left(\xi_{M}{ }^{-3}+\xi_{A}\right.$ $\left.{ }^{-3}\right)^{-1 /} \approx 0.57 \mu \mathrm{m} .{ }^{30}$ This comparison suggests that the more extreme subdiffusion in actin networks and composites compared to microtubules arises from the larger $R_{\text {coil }} \xi \xi$ ratio, as $R_{\text {coil }} \cdot \xi>1$ for actin and composites versus $R_{\text {coil }} \cdot \xi<1$ for microtubule networks. Further, in the equimolar composite network, $\xi_{A}$ is $\sim 2 x$ smaller than $\xi_{M}$ such that the environment the DNA encounters is much closer to a network of actin than microtubules. ${ }^{30}$ We plan to conduct future studies in which we vary the DNA size and protein concentration to further test this hypothesis.

Subdiffusion that depends on the ratio $a: \xi$ has been suggested to arise from caging effects, in which the crowded molecules get trapped in localized 'pockets' of the network for extended periods before hopping to new pockets. The distribution of trapping times results in subdiffusion over many decades in time..$^{4,7,50,51}$ Such caging and hopping behavior should also manifest itself as large heterogeneities in transport measured for different molecules in different regions of the network. ${ }^{4,50}$ Within this framework, more extreme subdiffusion (smaller $a$ ), resulting from more efficient caging, should be coupled with larger heterogeneities in transport. This phenomenon is in fact exactly what we see. Crowding by actin and composites leads to a high degree of heterogeneity in DNA transport compared to microtubule networks. Large-scale spatial heterogeneity is evident in the substantial error bars in $K$ and $a$ values for these systems.

To explore this heterogeneity phenomenon further, we evaluate the trajectories of single molecules as well as the range of $\tau(q)$ curves for different regions within each network (Fig. 5). As shown, in buffer conditions DNA exhibits minimal heterogeneities in single-molecule transport characteristics, and measurements of $\tau$ vs. $q$ from different regions of the sample all cluster together (Fig. 5A). Microtubule networks exhibit similar consistency among molecular trajectories, and the breadth of $\tau(q)$ values is not significantly larger than that for buffer conditions (Fig. 5B). Conversely, the distribution of $\tau$ values measured in different regions of the actin networks and composites spans an order of magnitude across nearly all lengthscales. Also, as evident in temporal color maps, there is a wide range of singlemolecule trajectories, from normal Brownian motion (albeit more restricted than in microtubules and buffer) to apparent caging in which the molecule appears nearly stationary over the entire $2 \mathrm{~s}$. This heterogeneity is exactly the reason that computing transport properties using only single-molecule or only ensemble methods becomes so difficult in crowded systems such as these and can lead to inaccurate or incomplete characterization of molecular dynamics.

We also recently demonstrated that the actin and microtubule networks under investigation display markedly different viscoelastic properties, arising from the $\sim 100$-fold difference in bending rigidity of actin compared to microtubules as well as the difference in mesh sizes. ${ }^{30}$ We found that microtubule networks stiffened in response to strain while actin networks and 
equimolar composites exhibited strain-softening, since actin can undergo bending fluctuations inaccessible to microtubules. Further, microtubule networks exhibited large heterogeneities in force response compared to actin and composites due to the larger mesh size and reduced mobility of microtubule networks. ${ }^{30,48,55}$ These results, together with our work here, suggest that more mobile and less rigid networks enhance the degree of subdiffusion.

Recent simulations have shown that the slow mobility of large crowders, which results in continuous temporal evolution of the crowding mesh, plays an important role in dictating anomalous subdiffusion of intruding particles. ${ }^{7}$ In particular, when the particle size is comparable to the mesh size of the crowders, only slowly moving crowders result in true subdiffusion over several decades in time. Conversely, immobile crowders resulted in transient subdiffusion, while freely diffusing crowders resulted in normal diffusion. Authors explain this phenomenon as arising from the particle motion being coupled to the crowding network motion such that they are transiently trapped in network voids but can traverse voids as they are rearranged over timescales comparable to the rearrangement time of the network. Similarly, we can understand our results as arising from the fact that actin networks and composites exhibit more pronounced and frequent fluctuations compared to relative stiff and immobile microtubule networks.

\section{Conclusions}

In conclusion, we have coupled recently-developed single-molecule and ensemble techniques (SMCT and SPIDDM) to elucidate the transport properties and conformational dynamics of large DNA molecules crowded by cytoskeletal networks. We span a wide range of spatiotemporal scales $(\sim 0.1-60 \mathrm{~s}, \sim 0.1-25 \mu \mathrm{m})$ to determine the relationship between single-molecule and network-spanning dynamics and link DNA dynamics to the properties of the surrounding network. We find that all cytoskeletal networks lead to DNA compaction, suppression of conformational fluctuations, slow and anomalous subdiffusion, and heterogeneous transport. However, the extent to which each of these phenomena occurs depends on the type(s) of filaments in the network. Actin induces the most extreme compaction, fluctuation suppression, transport heterogeneities, and subdiffusion, whereas microtubules have the least impact across all scales. Composites display a surprising scaledependent effect, with the presence of microtubules dictating single-molecule conformations and fluctuations, whereas actin filaments drive ensemble transport properties. This complex behavior highlights the importance of characterizing dynamics across wide-ranging scales to paint a complete picture of macromolecular transport in complex crowded environments. While the systems we study are specifically relevant to the periphery of cells, where networks of actin and microtubules sterically interact and where viral and therapeutic DNA must traverse, ${ }^{9,23}$ our presented platform offers a general and broadly applicable method. Our future work will focus on tackling the vast phase space of physiologically relevant system parameters by investigating the role of filament concentration and crosslinking, as well as DNA length and topology. However, our platform can be generally applied to a vast array of biologically relevant in vitro systems to elucidate and connect molecular-level fluctuations and ensemble transport properties. 


\section{Methods}

\section{Sample preparation:}

Double-stranded supercoiled DNA, $115 \mathrm{kbp}$ in length, is prepared via replication of bacterial artificial chromosome constructs in Escherichia coli followed by extraction and purification as described previously. ${ }^{56,57}$ Supercoiled DNA is converted to linear topology via treatment with the restriction enzyme MluI (New England Biolabs). ${ }^{56}$ For microscopy experiments, DNA is labeled with YOYO-I dye (ThermoFisher) at a basepair to dye ratio of 4:1. ${ }^{56}$ Cytoskeleton protein solutions are prepared following previously optimized and validated protocols. ${ }^{30}$ In short, rabbit skeletal actin monomers and/or porcine brain tubulin dimers (Cytoskeleton) are resuspended in an aqueous buffer consisting of 100mM PIPES, $2 \mathrm{mM}$ $\mathrm{MgCl}_{2}, 2 \mathrm{mM}$ EGTA, 2mM ATP, $1 \mathrm{mM}$ GTP and $5 \mu \mathrm{M}$ Taxol. ${ }^{30}$ ATP and GTP are required to polymerize actin and tubulin into protein filaments and Taxol is required to stabilize microtubules against depolymerization. $0.05 \%$ Tween is added to prevent surface interactions, and glucose $(0.9 \mathrm{mg} / \mathrm{ml})$, glucose oxidase $(0.86 \mathrm{mg} / \mathrm{ml})$, and catalase $(0.14$ $\mathrm{mg} / \mathrm{ml}$ ) are added to inhibit photobleaching. Labeled DNA is then added to protein solutions for a final concentration of (1) $0.025 \mu \mathrm{g} / \mathrm{ml}$ for single-molecule tracking experiments (Fig. 1, left) or (2) $20 \mu \mathrm{g} / \mathrm{ml}$ for DDM measurements (Fig. 1, right). Final solutions are pipetted into a sample chamber consisting of: (1) a glass slide and coverslip separated by double-sided tape (for SMCT) or (2) capillary tubing that is index-matched to water (for SPIDDM). Chambers are sealed with epoxy and incubated at $37^{\circ} \mathrm{C}$ for 30 minutes to form an entangled network of actin filaments and/or microtubules. Networks formed within both types of sample chambers are indistinguishable from one another (Fig. 2). Networks consist of either (i) only actin, (ii) only tubulin, or (iii) a 1:1 molar ratio of actin:tubulin, with the total protein concentration fixed at $11.4 \mu \mathrm{M}$. All three network types, fully characterized in Ref 30 , consist of randomly oriented entangled filaments with minimal bundling and no phase separation between actin and microtubules (Fig. 2). ${ }^{30}$ The length distributions for actin and microtubules in networks were measured to be $8.7 \pm 2.8$ and $17.8 \pm 9.7 \mu \mathrm{m} .{ }^{30}$

\section{Imaging:}

For SMCT, DNA is imaged with a Nikon Eclipse A1R epifluorescence microscope with a $60 x$ objective and QImaging CCD camera. Single DNA molecules are imaged and tracked at a frame rate of $10 \mathrm{fps}$ for 20 frames (Fig. 1). All data presented are from an ensemble of $\sim 200$ molecules imaged in 2-4 different samples for each condition. Custom-written inhouse software (MATLAB) is used to measure and track the major axis length $\left(R_{\max }\right)$ and minor axis length $\left(R_{\min }\right)$ of each molecule in each frame. We calculate an effective coil size $\left(R_{c o i l}\right)$ from the major and minor axis length measurements via

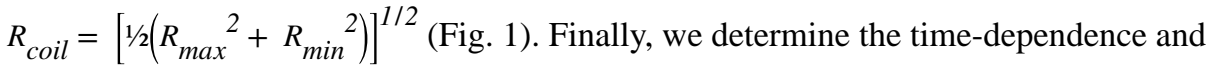
lengthscale of conformational fluctuations by calculating the fluctuation length $L(t)=\left\langle\left|R_{\max }(t)-R_{\max }(0)\right|>\right.$ for a given lag time $t$, which quantifies the lengthscale over which single molecules fluctuate between different conformational states and the timescale over which fluctuations relax to steady-state values. These acquisition and analysis methods, depicted in Fig. 1, have been thoroughly described and validated previously. 3,6,32,58 
For DDM measurements, samples are imaged with a home-built light-sheet microscope with a $10 \times 0.25 \mathrm{NA}$ excitation objective, $20 \times 0.5 \mathrm{NA}$ water-dipping imaging objective, and Andor Zyla 4.2 sCMOS camera. Ensembles of DNA molecules are imaged in ROIs of $128 \times 1024$ pixels $(24.2 \times 193.5 \mu \mathrm{m})$ or $256 \times 1024$ pixels $(48.4 \times 193.5 \mu \mathrm{m})$ for buffer and protein conditions respectively. Sample videos are taken at 29 fps for 5000 frames (Fig. 1). All data presented in Figures 4 and 5 are from imaging 6 different locations within each sample and 2-4 samples for each condition. For DDM analysis, images are broken into $128 \times 128$ or $256 \times 256$ pixel ROIs for buffer and protein conditions respectively and then analyzed using established DDM routines. ${ }^{31}$ Image structure functions $D(q, t)$, where $q$ is the spatial frequency and $t$ is the lag time, are fit to a stretched exponential

$D(q, t)=A(q)\left[1-\exp \{-t / \tau(q)\}^{\beta(q)}\right]+B(q)$ where $A(q)$ depends on the optical properties of the sample and microscope, $\beta(q)$ is the system-dependent stretching exponent, and $B(q)$ is a background parameter. We found that a stretched exponential best fit our data and allowed us to use a single function to fit $D(q, t)$ in all conditions. Prior studies of diffusion in complex environments using DDM have also employed a stretched exponential to fit $D(q, t) .{ }^{59}$

$\tau(q)$ values, which represent the characteristic decay time of density fluctuations at each spatial frequency, are determined from fits to $D(q, t)$ (Fig. 1), and transport coefficients $K$ are then calculated via the relation $\tau(q)=\left(K q^{2}\right)^{-1 / a} .31,33,59$ For a system undergoing normal Brownian diffusion, in which the MSD scales linearly with time, $K$ is equivalent to the diffusion coefficient $D$ and $a=1$. In calculating the $K$ and a values, we only use data where we could reliably fit the image structure function and where we observe a plateau in that function. This resulted in the following range of useable $q$ values for each condition: $0.5-$ $4.0 \mu \mathrm{m}^{-1}$ (buffer, microtubules), $1.9-4.0 \mu \mathrm{m}^{-1}$ (actin), and $2.1-4.0 \mu \mathrm{m}^{-1}$ (composite).

\section{Acknowledgements}

This work was performed with funding from NIH-NIGMS award no. R15GM123420 to RMR-A and RM, AFOSR award no. FA9550-17-1-0249 to RMR-A, and ACS PRF award no. 57326-UNI10 to RM. The authors would also like to thank R. Dotterweich for her contributions to the initial stages of this project.

\section{References}

1. Ellis RJ Macromolecular crowding: an important but neglected aspect of the intracellular environment. Curr. Opin. Struct. Biol. 11, 114-119 (2001). [PubMed: 11179900]

2. Nakano S, Miyoshi D \& Sugimoto N Effects of Molecular Crowding on the Structures, Interactions, and Functions of Nucleic Acids. Chem. Rev. 114, 2733-2758 (2014). [PubMed: 24364729]

3. Gorczyca SM, Chapman CD \& Robertson-Anderson RM Universal scaling of crowding-induced DNA mobility is coupled with topology-dependent molecular compaction and elongation. Soft Matter 11, 7762-7768 (2015). [PubMed: 26303877]

4. Höfling F \& Franosch T Anomalous transport in the crowded world of biological cells. Rep. Prog. Phys. 76, 046602 (2013). [PubMed: 23481518]

5. Zinchenko A DNA conformational behavior and compaction in biomimetic systems: Toward better understanding of DNA packaging in cell. Adv. Colloid Interface Sci. 232, 70-79 (2016). [PubMed: 26976700]

6. Mardoum WM, Gorczyca SM, Regan KE, Wu T-C \& Robertson-Anderson RM Crowding Induces Entropically-Driven Changes to DNA Dynamics That Depend on Crowder Structure and Ionic Conditions. Front. Phys. 6, (2018). 
7. Sentjabrskaja T et al. Anomalous dynamics of intruders in a crowded environment of mobile obstacles. Nat. Commun. 7, 11133 (2016). [PubMed: 27041068]

8. Trovato F \& Tozzini V Diffusion within the Cytoplasm: A Mesoscale Model of Interacting Macromolecules. Biophys. J. 107, 2579-2591 (2014). [PubMed: 25468337]

9. Miyoshi D \& Sugimoto N Molecular crowding effects on structure and stability of DNA. Biochimie 90, 1040-1051 (2008). [PubMed: 18331845]

10. Tan C, Saurabh S, Bruchez MP, Schwartz R \& LeDuc P Molecular crowding shapes gene expression in synthetic cellular nanosystems. Nat. Nanotechnol. 8, 602-608 (2013). [PubMed: 23851358]

11. Szymanski J \& Weiss M Elucidating the origin of anomalous diffusion in crowded fluids. Phys. Rev. Lett. 103, 038102 (2009). [PubMed: 19659323]

12. Dauty E \& Verkman AS Molecular crowding reduces to a similar extent the diffusion of small solutes and macromolecules: measurement by fluorescence correlation spectroscopy. J. Mol. Recognit. JMR 17, 441-447 (2004). [PubMed: 15362103]

13. Ziębacz N, Wieczorek SA, Kalwarczyk T, Fiałkowski M \& Hołyst R Crossover regime for the diffusion of nanoparticles in polyethylene glycol solutions: influence of the depletion layer. Soft Matter 7, 7181-7186 (2011).

14. Cheng Y, Prud'homme RK \& Thomas JL Diffusion of Mesoscopic Probes in Aqueous Polymer Solutions Measured by Fluorescence Recovery after Photobleaching. Macromolecules 35, 81118121 (2002).

15. Dauty E \& Verkman AS Actin cytoskeleton as the principal determinant of size-dependent DNA mobility in cytoplasm: a new barrier for non-viral gene delivery. J. Biol. Chem. 280, 7823-7828 (2005). [PubMed: 15632160]

16. Lukacs GL et al. Size-dependent DNA Mobility in Cytoplasm and Nucleus. J. Biol. Chem. 275, 1625-1629 (2000). [PubMed: 10636854]

17. Jones JJ, van der Maarel JRC \& Doyle PS Effect of Nanochannel Geometry on DNA Structure in the Presence of Macromolecular Crowding Agent. Nano Lett. 11, 5047-5053 (2011). [PubMed: 21988280]

18. Zorrilla S, Hink MA, Visser AJWG \& Lillo MP Translational and rotational motions of proteins in a protein crowded environment. Biophys. Chem. 125, 298-305 (2007). [PubMed: 17007994]

19. Regner BM et al. Anomalous diffusion of single particles in cytoplasm. Biophys. J. 104, 16521660 (2013). [PubMed: 23601312]

20. Rashid R, Chee SML, Raghunath M \& Wohland T Macromolecular crowding gives rise to microviscosity, anomalous diffusion and accelerated actin polymerization. Phys. Biol. 12, 034001 (2015). [PubMed: 25927668]

21. Weiss M, Elsner M, Kartberg F \& Nilsson T Anomalous Subdiffusion Is a Measure for Cytoplasmic Crowding in Living Cells. Biophys. J. 87, 3518-3524 (2004). [PubMed: 15339818]

22. Nakano S \& Sugimoto N Model studies of the effects of intracellular crowding on nucleic acid interactions. Mol. Biosyst. 13, 32-41 (2016). [PubMed: 27819369]

23. Huber F, Boire A, López MP \& Koenderink GH Cytoskeletal crosstalk: when three different personalities team up. Curr. Opin. Cell Biol. 32, 39-47 (2015). [PubMed: 25460780]

24. Gardel ML, Kasza KE, Brangwynne CP, Liu J \& Weitz DA Chapter 19: Mechanical response of cytoskeletal networks. Methods Cell Biol. 89, 487-519 (2008). [PubMed: 19118688]

25. Vaughan EE, DeGiulio JV \& Dean DA Intracellular trafficking of plasmids for gene therapy: mechanisms of cytoplasmic movement and nuclear import. Curr. Gene Ther. 6, 671-681 (2006). [PubMed: 17168698]

26. Kikumoto M, Kurachi M, Tosa V \& Tashiro H Flexural Rigidity of Individual Microtubules Measured by a Buckling Force with Optical Traps. Biophys. J. 90, 1687-1696 (2006). [PubMed: 16339879]

27. Rodriguez OC et al. Conserved microtubule-actin interactions in cell movement and morphogenesis. Nat. Cell Biol. 5, 599-609 (2003). [PubMed: 12833063]

28. Palmer A, Xu J, Kuo SC \& Wirtz D Diffusing wave spectroscopy microrheology of actin filament networks. Biophys. J. 76, 1063-1071 (1999). [PubMed: 9916038] 
29. Sanabria H \& Waxham MN Transient Anomalous Subdiffusion: Effects of Specific and Nonspecific Probe Binding with Actin Gels. J. Phys. Chem. B 114, 959-972 (2010). [PubMed: 20038146]

30. Ricketts SN, Ross JL \& Robertson-Anderson RM Co-Entangled Actin-Microtubule Composites Exhibit Tunable Stiffness and Power-Law Stress Relaxation. Biophys. J. 115, 1055-1067 (2018). [PubMed: 30177441]

31. Wulstein DM, Regan KE, Robertson-Anderson RM \& McGorty R Light-sheet microscopy with digital Fourier analysis measures transport properties over large field-of-view. Opt. Express 24, 20881-20894 (2016). [PubMed: 27607692]

32. Chapman CD, Gorczyca S \& Robertson-Anderson RM Crowding Induces Complex Ergodic Diffusion and Dynamic Elongation of Large DNA Molecules. Biophys. J. 108, 1220-1228 (2015). [PubMed: 25762333]

33. Cerbino R \& Trappe V Differential Dynamic Microscopy: Probing Wave Vector Dependent Dynamics with a Microscope. Phys. Rev. Lett. 100, 188102 (2008). [PubMed: 18518417]

34. Giavazzi F, Brogioli D, Trappe V, Bellini T \& Cerbino R Scattering information obtained by optical microscopy: differential dynamic microscopy and beyond. Phys. Rev. E Stat. Nonlin. Soft Matter Phys. 80, 031403 (2009). [PubMed: 19905112]

35. Reufer M, Martinez VA, Schurtenberger P \& Poon WCK Differential Dynamic Microscopy for Anisotropic Colloidal Dynamics. Langmuir 28, 4618-4624 (2012). [PubMed: 22324390]

36. Giavazzi F \& Cerbino R Digital Fourier microscopy for soft matter dynamics. J. Opt. 16, 083001 (2014).

37. Kuhn W Über die Gestalt fadenförmiger Moleküle in Lösungen. Kolloid-Z. 68, 2-15 (1934).

38. Haber C, Ruiz SA \& Wirtz D Shape anisotropy of a single random-walk polymer. Proc. Natl. Acad. Sci. 97, 10792-10795 (2000). [PubMed: 10984514]

39. Lerman LS A transition to a compact form of DNA in polymer solutions. Proc. Natl. Acad. Sci. U. S. A. 68, 1886-1890 (1971). [PubMed: 5288774]

40. Vasilevskaya VV, Khokhlov AR, Matsuzawa Y \& Yoshikawa K Collapse of single DNA molecule in poly(ethylene glycol) solutions. J. Chem. Phys. 102, 6595-6602 (1995).

41. Banks DS \& Fradin C Anomalous Diffusion of Proteins Due to Molecular Crowding. Biophys. J. 89, 2960-2971 (2005). [PubMed: 16113107]

42. Kang H, Toan NM, Hyeon C \& Thirumalai D Unexpected Swelling of Stiff DNA in a Polydisperse Crowded Environment. J. Am. Chem. Soc. 137, 10970-10978 (2015). [PubMed: 26267166]

43. Zinchenko AA \& Yoshikawa $\mathrm{K} \mathrm{Na}$ + shows a markedly higher potential than $\mathrm{K}+$ in DNA compaction in a crowded environment. Biophys. J. 88, 4118-4123 (2005). [PubMed: 15778438]

44. Zhang C, Shao PG, van Kan JA \& van der Maarel JRC Macromolecular crowding induced elongation and compaction of single DNA molecules confined in a nanochannel. Proc. Natl. Acad. Sci. U. S. A. 106, 16651-16656 (2009). [PubMed: 19805352]

45. Zhang $\mathrm{C}$ et al. Nanouidic Compaction of DNA by Like-Charged Protein. J. Phys. Chem. B 116, 3031-3036 (2012). [PubMed: 22320240]

46. Biswas N et al. Phase separation in crowded micro-spheroids: DNA-PEG system. Chem. Phys. Lett. 539-540, 157-162 (2012).

47. van't Hoff JH The Role of Osmotic Pressure in the Analogy Between Solutions and Gases. Z. Für Phys. Chem. 481-508 (1887).

48. Gittes F, Mickey B, Nettleton J \& Howard J Flexural rigidity of microtubules and actin filaments measured from thermal fluctuations in shape. J. Cell Biol. 120, 923-934 (1993). [PubMed: 8432732]

49. Bustamante C, Smith SB, Liphardt J \& Smith D Single-molecule studies of DNA mechanics. Curr. Opin. Struct. Biol. 10, 279-285 (2000). [PubMed: 10851197]

50. Wong IY et al. Anomalous Diffusion Probes Microstructure Dynamics of Entangled F-Actin Networks. Phys. Rev. Lett. 92, (2004).

51. Nath P et al. Dynamics of Nanoparticles in Entangled Polymer Solutions. Langmuir 34, 241-249 (2018). [PubMed: 29192503] 
52. Lin Y-C, Koenderink GH, MacKintosh FC \& Weitz DA Viscoelastic Properties of Microtubule Networks. Macromolecules 40, 7714-7720 (2007).

53. Schmidt CF, Baermann M, Isenberg G \& Sackmann E Chain dynamics, mesh size, and diffusive transport in networks of polymerized actin: a quasielastic light scattering and microfluorescence study. Macromolecules 22, 3638-3649 (1989).

54. Gardel ML, Valentine MT, Crocker JC, Bausch AR \& Weitz DA Microrheology of Entangled FActin Solutions. Phys. Rev. Lett. 91, (2003).

55. Fakhri N, MacKintosh FC, Lounis B, Cognet L \& Pasquali M Brownian Motion of Stiff Filaments in a Crowded Environment. Science 330, 1804-1807 (2010). [PubMed: 21205665]

56. Robertson RM, Laib S \& Smith DE Diffusion of isolated DNA molecules: Dependence on length and topology. Proc. Natl. Acad. Sci. 103, 7310-7314 (2006). [PubMed: 16648255]

57. Laib S, Robertson RM \& Smith DE Preparation and Characterization of a Set of Linear DNA Molecules for Polymer Physics and Rheology Studies. Macromolecules 39, 4115-4119 (2006).

58. Chapman CD, Shanbhag S, Smith DE \& Robertson-Anderson RM Complex effects of molecular topology on diffusion in entangled biopolymer blends. Soft Matter 8, 9177 (2012).

59. Jacob JDC, He K, Retterer ST, Krishnamoorti R \& Conrad JC Diffusive dynamics of nanoparticles in ultra-confined media. Soft Matter 11, 7515-7524 (2015). [PubMed: 26278883] 

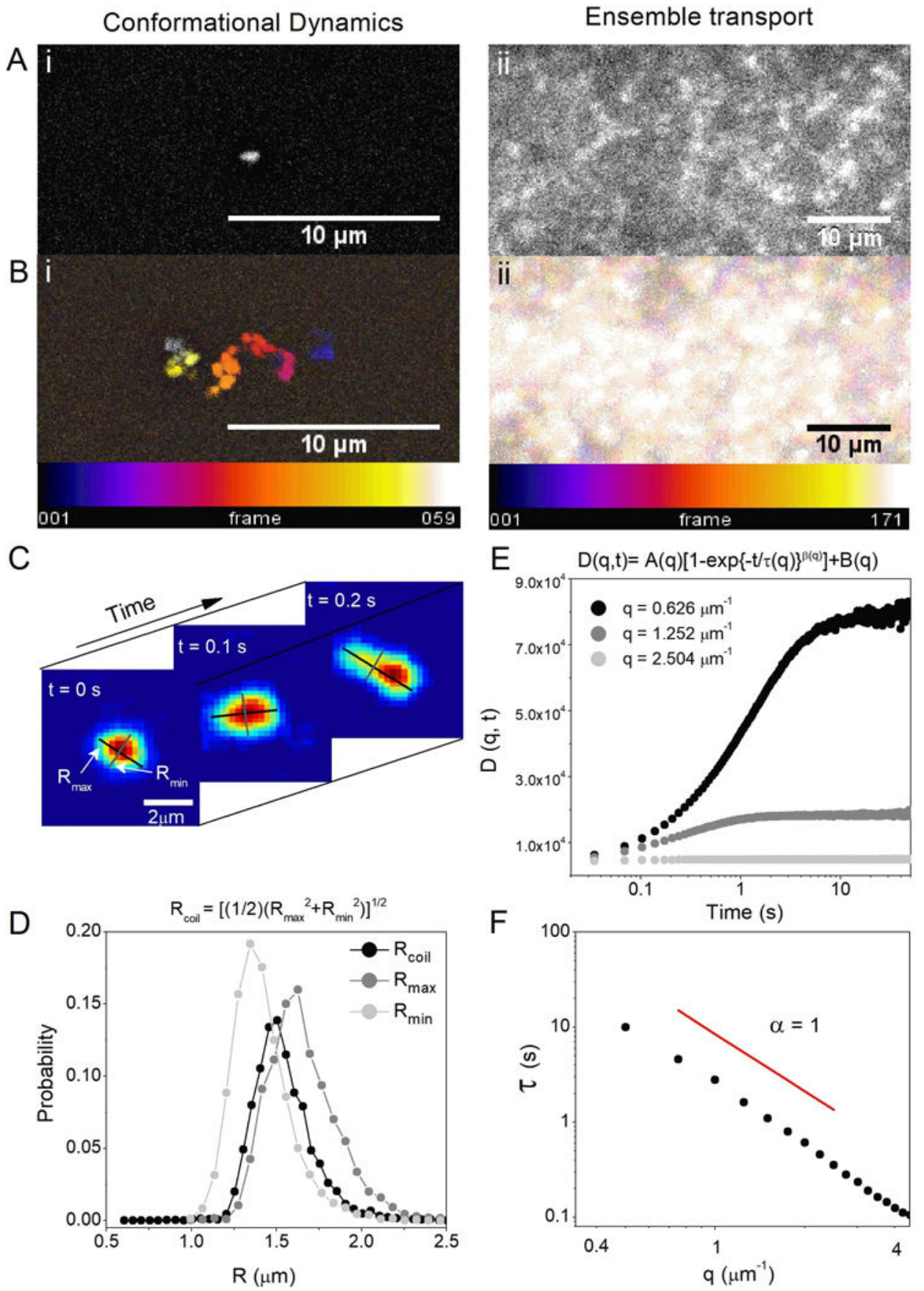

Figure 1. Coupling SMCT with SPIDDM enables robust measurements of macromolecular conformations and transport properties over a wide spatiotemporal range.

(A) High-resolution epifluorescence imaging resolves conformations of single DNA molecules (i) while LSM imaging over large field-of-views captures ensemble DNA dynamics (ii). (B) Temporal color maps of videos of DNA acquired at 10 fps using epifluorescence (i) and 29 fps using LSM (ii) display the different size scales between motion at the single-molecule and ensemble levels, as well as the scopes of transport being measured. The combined method allows for resolution of single-molecule shape dynamics and trajectories (i) as well as statistically robust ensemble transport properties (ii) where 
single-molecule motion is beyond spatial resolution. (C) SMCT measures major and minor axis lengths $R_{\max }$ and $R_{\min }$ of each molecule for each frame of each video. (D) Distributions of $R_{\max }$ and $R_{\min }$ for the entire ensemble of molecules and frames, as well as the distribution of coil sizes $R_{\text {coil }}=\left[1 / 2\left(R_{\max }{ }^{2}+R_{\text {min }}{ }^{2}\right)\right]^{1 / 2}$ quantify the population of conformational sizes and shapes accessed by molecules. (E) DDM algorithms compute the radial average of Fourier transforms of differences of images separated by a given lag time. This analysis provides the image structure function $D(q, t)$, which is fit to a stretched exponential to extract decay times for each accessible spatial frequency. The stretching exponent is $\sim 1.0$ in buffer conditions but smaller in protein networks. (F) To describe ensemble diffusion properties, the characteristic decay time $\tau$ for a given $q$ is fit to a power-law relation $\tau=\left(K q^{2}\right)^{-1 / a}$ where $K$ is the transport coefficient and $a$ is the diffusive scaling exponent. All data shown is for DNA in buffer conditions (see text for details). 


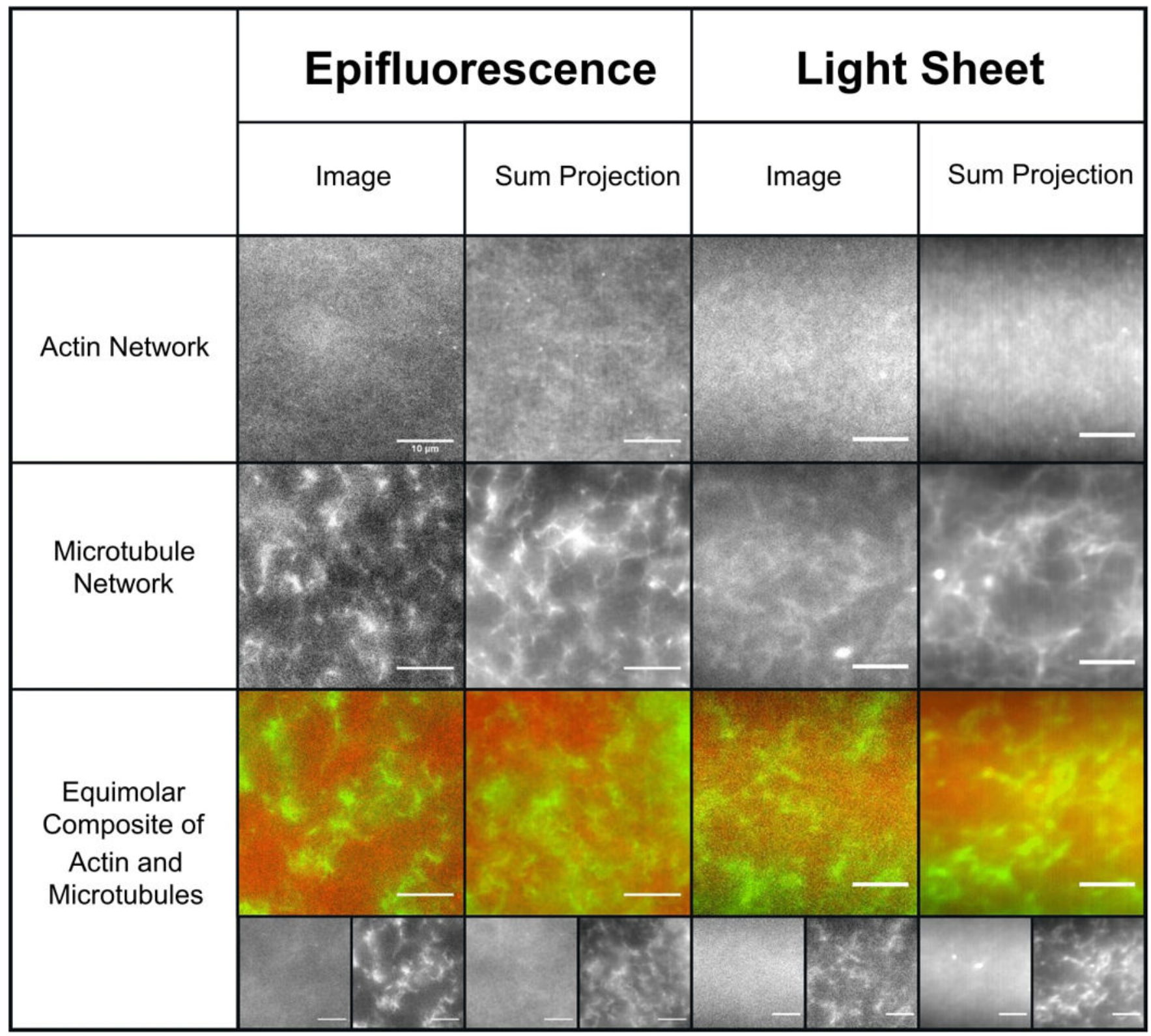

Figure 2. In vitro cytoskeleton networks exhibit robust characteristics and structure. Representative images (left) and sum projections of videos (right) taken using the epifluorescence and light-sheet microscopy methods used for SMCT and SPIDDM measurements. All scale bars represent $10 \mu \mathrm{m}$. As shown, networks formed in the respective sample chambers are nearly indistinguishable from one another. Actin networks have a smaller mesh size than microtubule networks, exhibited as more uniformity at the microscale. Microtubules form more rigid structures, exhibited as higher contrast projections compared to actin. In composites, actin and microtubules are well-mixed and sterically interacting with no large-scale phase separation. In all images, actin and tubulin are labeled with Alexa-488 (1:5 ratio labeled:unlabeled monomers) and rhodamine (1:35 labeled:unlabeled dimer ratio).Videos are all 400 frames acquired at $40 \mathrm{fps}$. Though images shown have labeled filaments, experiments are carried out with unlabeled protein only. 

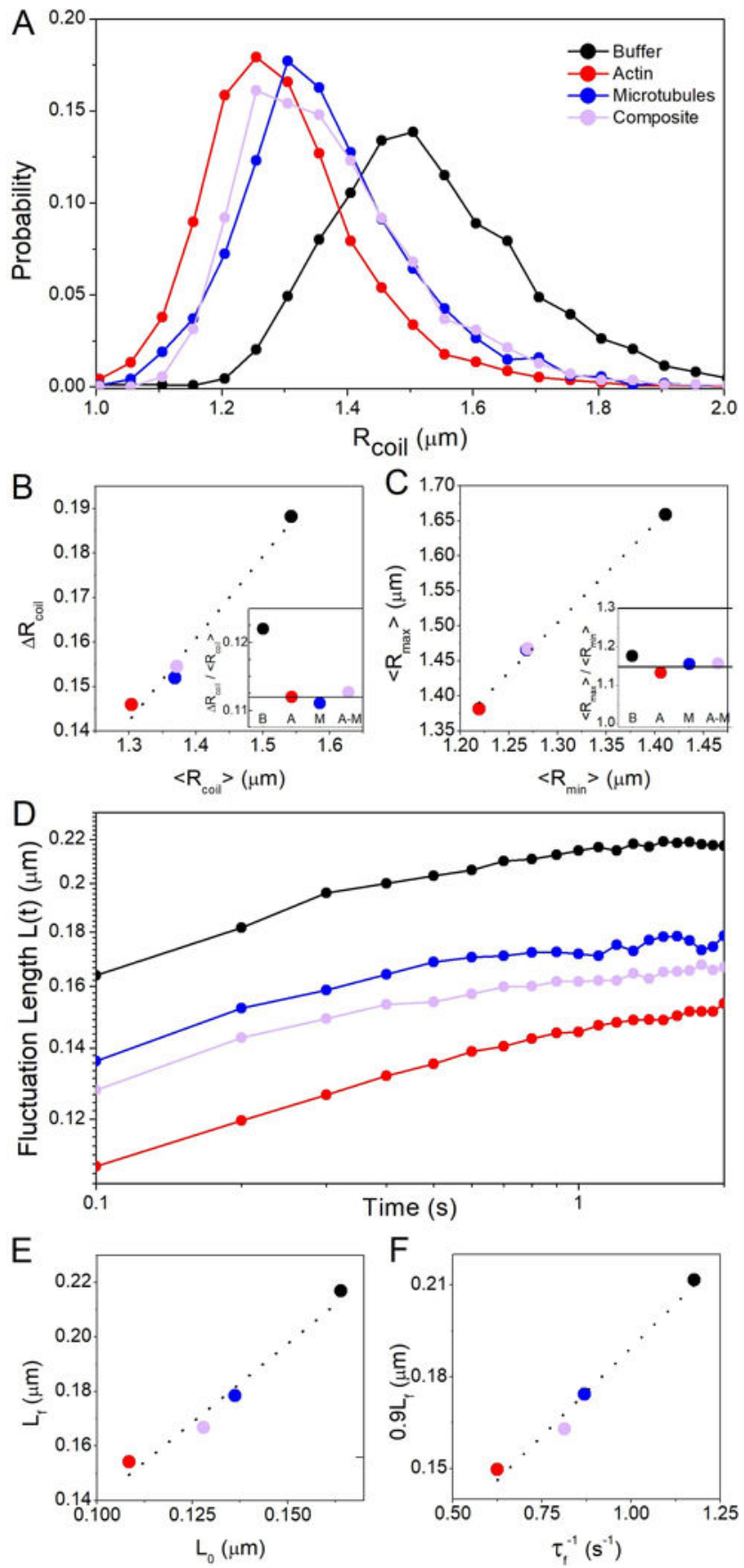

Figure 3. Actin networks induce the most pronounced compaction of DNA and slowing of conformational fluctuations.

(A) Probability distributions of DNA coil sizes $R_{\text {coil }}$ show network-dependent compaction of DNA. Distributions for microtubules and composites exhibit significant overlap while actin induces more pronounced compaction (distribution shifted to the left). (B) Standard deviation of coil size distribution $\Delta R_{\text {coil }}$ versus mean coil size $\left\langle R_{\text {coil }}\right\rangle$ with dashed line to guide the eye. Inset shows the ratio $\Delta R_{\text {coil }}\left\langle R_{\text {coil }}\right\rangle$ for buffer (B), actin (A), microtubules (M), composites (A-M). The horizontal line represents the average of the network values, showing that crowding leads to tighter distributions of coil sizes. (C) Mean major axis length 
$\left\langle R_{\max }\right\rangle$ versus minor axis length $\left\langle R_{\min }\right\rangle$ with dashed line to guide the eye. Inset shows the ratio $\left\langle R_{\max }\right\rangle:\left\langle R_{\min }\right\rangle$, with the horizontal line representing the average of the network values. (D) Fluctuation length $L(t)$ vs time shows that DNA fluctuates between different conformational states with fluctuation rates and lengthscales dependent on crowding conditions. (E) The final fluctuation length $L_{f}$ vs initial length $L_{O}$ with dashed line indicating the linear relation $L_{f}=1.16 L_{0}$. (F) $90 \%$ of $L_{f}$ vs the rate $\tau_{f}^{-1}$ at which $L(t)$ reaches $0.9 L_{f}$ with dashed line showing the linear relation $0.9 L_{f}=0.12 \tau_{f}^{-1}$. As shown in (E,F), actin suppresses the lengthscale and rate of fluctuations most appreciably while composites exhibit suppression intermediate between that of actin and microtubule networks. 

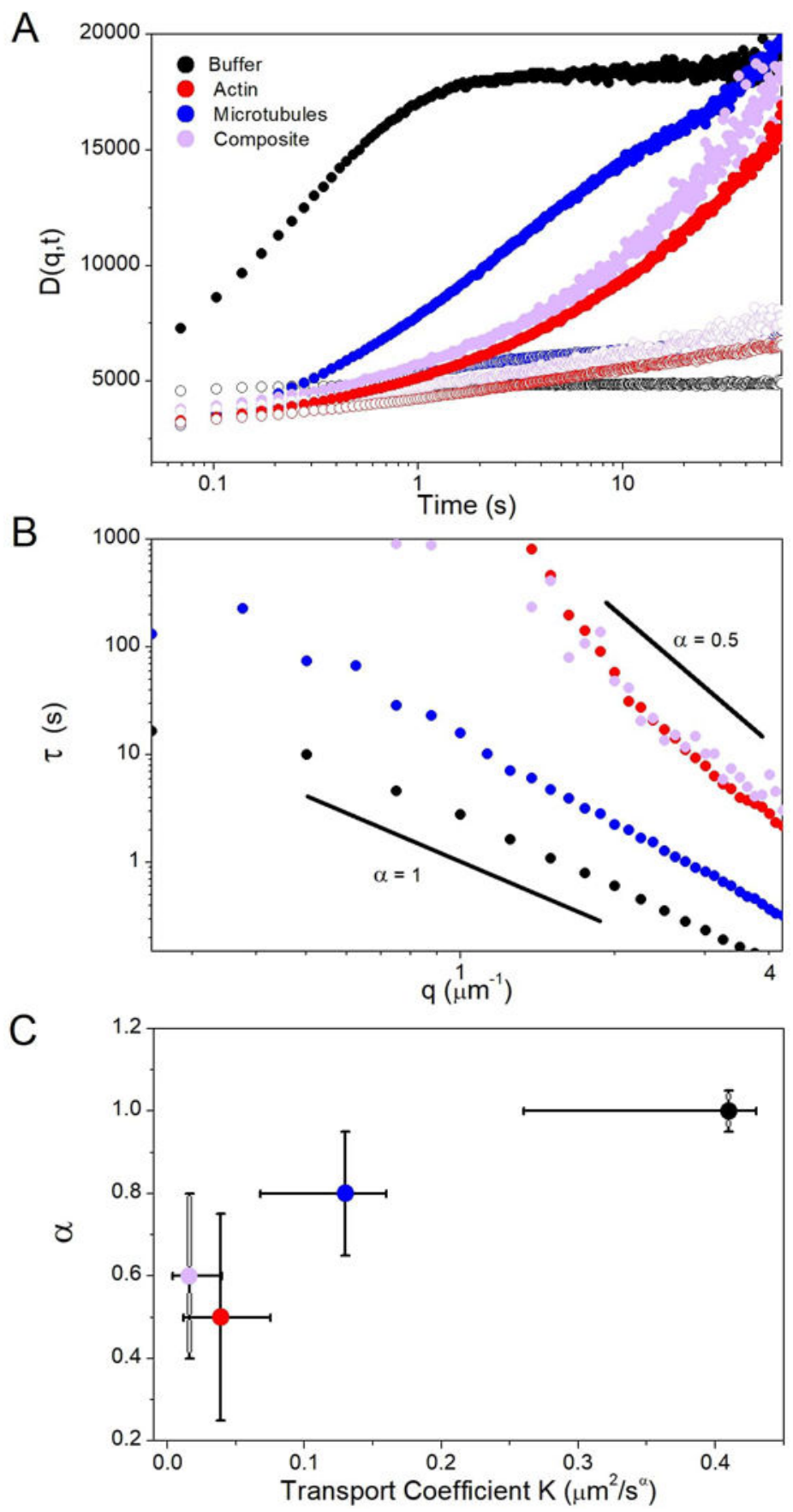

Figure 4. Actin drives extreme slowing of ensemble transport and subdiffusive dynamics of DNA in cytoskeleton networks.

(A) DDM image structure functions $D(q, t)$ vs time for two different spatial frequencies: $q=$ $1.25 \mu^{-1}$ (filled) and $q=2.50 \mu^{-1}$ (open). DNA transport exhibits similar slow and anomalous behavior in actin and composites while microtubule networks have a reduced impact on transport. (B) The characteristic decay time $\tau$ for each spatial frequency $q$, determined via fits to $D(q, t)$ (see Methods, Fig. 1). $\tau(q)$ exhibits power-law behavior that is fit to $\tau=\left(K q^{2}\right)^{-1 / a}$. Solid black lines show $a=1$ (normal diffusion) and $a=0.5$ 
(subdiffusion). (C) Transport coefficient $K$ versus $a$ for each system. Error bars represent the range in $K$ and $a$ among at least 20 different regions in each of the conditions. 

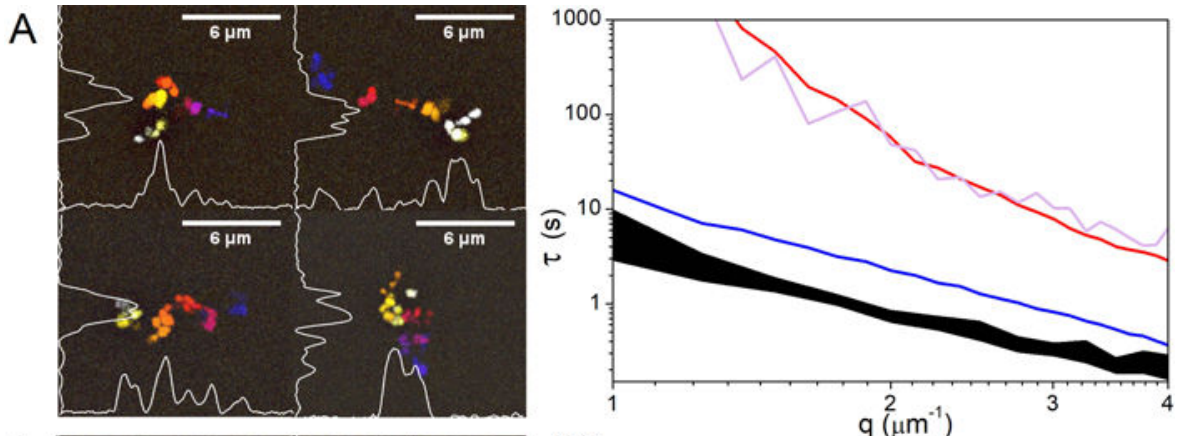

B
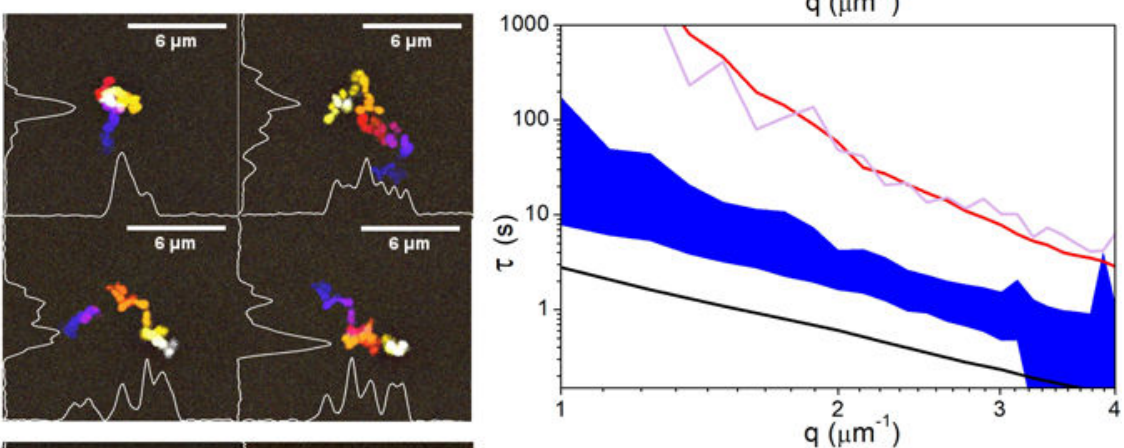

C
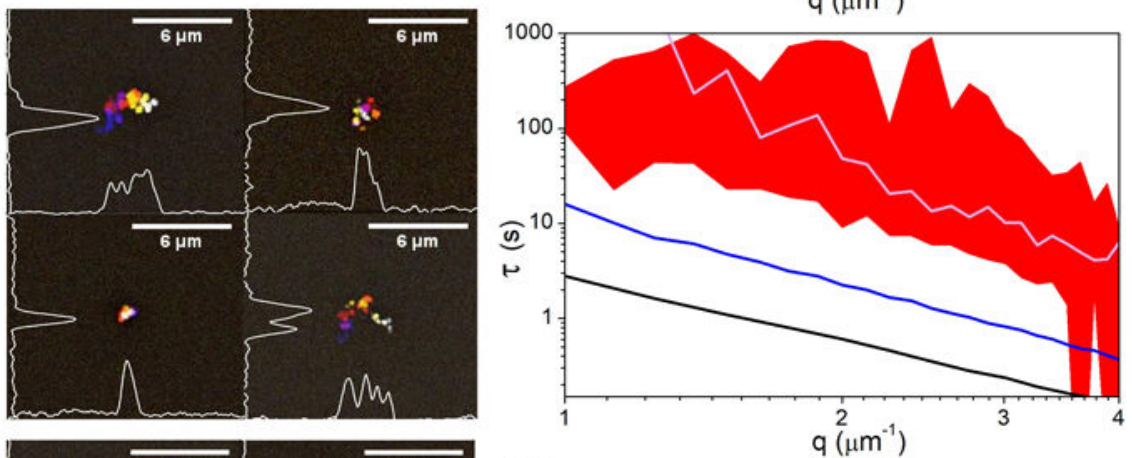

D
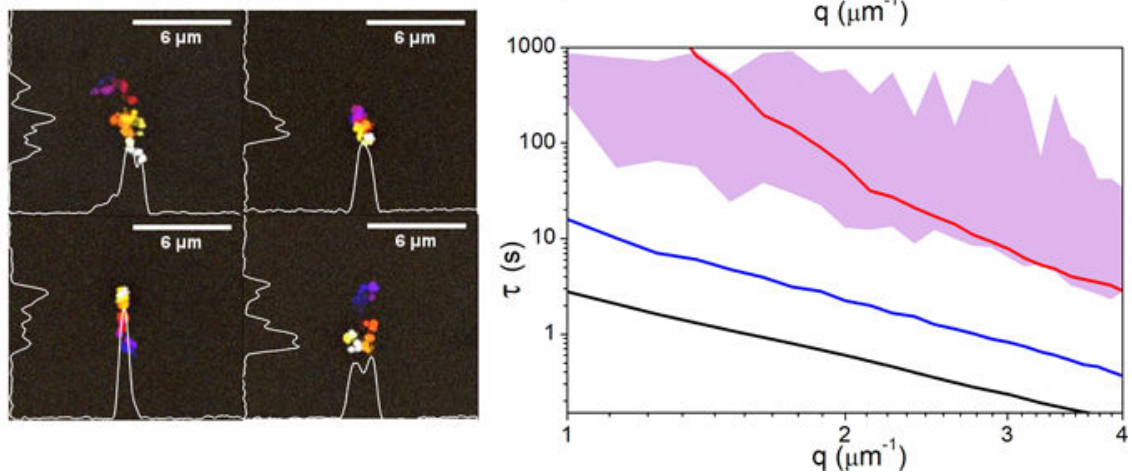

Figure 5. DNA displays heterogeneous transport properties in cytoskeletal networks at both single-molecule and ensemble scales.

(Left) Temporal color maps showing sample trajectories of single DNA molecules diffusing in buffer conditions (A), actin (B), microtubules (C) and composites (D). (Right)

Characteristic decay times $(\tau)$ versus spatial frequency $(q)$ for each condition. Filled regions indicate the range in data measured in at least 20 different regions of the network. Transport in buffer conditions (A) shows minimal heterogeneity in both single-molecule trajectories and the ensemble distribution of dynamics. Transport in actin and composites (C,D) exhibit a high degree of heterogeneity at both scales. Molecular trajectories are highly varied and 
the range in ensemble data is quite broad for both systems compared to buffer conditions. Conversely, transport on both scales exhibits much more uniformity, comparable to that in buffer conditions, when crowded by microtubules (B). 